\title{
Effects of Hesperetin on the Serum Superoxide Dismutase, Glutathione Peroxidase, and Malondialdehyde Levels and Renal-Histopathological Alterations Following Skeletal Muscle Ischemia Reperfusion in Male Rats
}

\author{
Hamed Ashrafzadeh Takhtfooladi ${ }^{1}$, Pejman Mortazavi ${ }^{1 *}$, Ahmad Asghari ${ }^{2}$, Fariborz Moayer ${ }^{3}$
}

\begin{abstract}
Objectives: The aim of the current study was to assess the effects of hesperetin on damage to kidneys as remote organs following skeletal muscle ischemia-reperfusion (IR) in rats.

Materials and Methods: In general, 30 male Wistar rats were randomized and placed into sham, IR, hesperetin, dimethyl sulfoxide (DMSO), and IR+hesperetin groups. The rats in the hesperetin and IR+hesperetin groups received a $50 \mathrm{mg} / \mathrm{kg}$ dose of hesperetin dissolved in DMSO intraperitoneally. In the IR+hesperetin group, hesperetin was injected exactly prior to reperfusion. To induce skeletal muscle ischemia, the femoral artery was clamped for two hours. Following a 24-hour period of reperfusion, the samples of blood were collected for renal function tests and oxidative stress measurements. Next, the rats were euthanized, and histological analyses were conducted on their removed kidneys.

Results: Based on the results, urea and creatinine serum levels were significantly higher in the IR group $(P<0.05)$ whereas they significantly reduced following treatment with hesperetin $(P<0.05)$. The concentration of malondialdehyde $(P<0.05)$ increased for the IR group while those of superoxide dismutase $(P<0.05)$ and glutathione peroxidase $(P<0.05)$ activities were lower than the other groups. The analysis of renal tissues in the IR group showed glomerular necrosis, degeneration, and necrosis of the tubular epithelium, protein casts, interstitial edema, and inflammation. Finally, the degree of renal injury was significantly ameliorated $(P<0.05)$ in rats treated with hesperetin.

Conclusions: Overall, the results indicated that in rats, hesperetin could reduce renal injury that has been induced through skeletal muscle IR.

Keywords: Hesperetin, Skeletal muscle, Ischemia reperfusion, Renal function
\end{abstract}

\section{Introduction}

Ischemia-reperfusion (IR) to the extremities is exemplified by greater creatine kinase serum levels, metabolic acidosis, myoglobinuria, and hyperkalemia with an intracellular potassium deficiency, as well as the release of free radicals (1). During reperfusion, the oxygenated blood entering the ischemic tissue carries many free radicals, which subsequently leads to further tissue damage (2).

The dysfunction of remote organs occurs only after reperfusion, implying that cellular mediators locally produced in the limb were responsible for mediating injuries to remote organs (3). Kidneys are particularly important in remote organ consequences following skeletal muscle IR. Taking this into account, research has focused on the renal function and histological lesions in animal models after hindlimb IR (4).

Natural products and their derivatives exhibit efficient anti-oxidative and anti-inflammatory activities (5).
A number of pharmacological properties have been attributed to flavonoids, which act as free radical scavengers (6).

Large amounts of hesperetin, as a bioflavonoid, can be isolated from the rinds of some Citrus species (7). It has been shown that hesperetin is a potent anti-inflammatory, antioxidant, anti-apoptotic, antimicrobial, antifungal, and antiviral agent with lipid-lowering and insulin-sensitizing activities, therefore, it has therapeutic effects against various diseases $(8,9)$.

Despite various applications of hesperetin, there is no information about its potential role in reducing damage to remote organs following IR. The results of our study would help clarify the potential importance of hesperetin in this situation of oxidative stress. With this in mind and based on the literature review, it was hypothesized that hesperetin might have a protective effect on renal remote injury in rats. Thus, the current study primarily

Received 16 July 2020, Accepted 2 October 2020, Available online 26 October 2020

'Department of Pathobiology, Science and Research Branch, Islamic Azad University, Tehran, Iran.

${ }^{2}$ Department of Clinical Science, Science and Research Branch, Islamic Azad University, Tehran, Iran.

${ }^{3}$ Department of Pathobiology, School of Veterinary Medicine, Karaj Branch, Islamic Azad University, Alborz, Iran.

*Corresponding Author: Pejman Mortazavi, Email: pejmanmortazavi99@gmail.com 
Key Messages

- Ischemia-reperfusion injury (IRI) results in a local injury and can also cause damage to remote organs.

- There is no ideal drug preserving the remote organs from the adverse effects of IRI.

- Hesperetin is a product of citrus fruits that is safe and cheap. It has therapeutic effects against various diseases.

- In our study, hesperetin reduced the renal oxidative stress and histopathological injury scores from skeletal muscle IRI.

- The results of this study showed hesperetin can diminish the injuries in the renal remote organ. To the best of our knowledge, it is the first study to address this beneficial effect.

aimed to examine the influence of hesperetin on renal injury after skeletal muscle IR by conducting biochemical, histopathological, and functional analyses in an animal model.

\section{Materials and Methods}

Hesperetin Preparation

Hesperetin (Sigma-Aldrich Chemical Company, PCode: 1002592101, Product of China) was freshly prepared by dissolving the powder in dimethyl sulfoxide (DMSO; Sigma-Aldrich, USA) for intraperitoneal administration.

\section{Experimental Groups}

Thirty Wistar male rats (220-250 g) from the same colony were included in this study that were deemed clinically healthy. The animals were held in individual cages at room temperature $\left(22 \pm 2^{\circ} \mathrm{C}\right)$ in a $12: 12$ hour light/dark cycle. In their cage, the animals had open access to both rodent food and water at all times. The rats were randomly assigned to five distinct groups each consisting of six animals. Group I (sham group) underwent all study operative procedures, excluding the arterial occlusion and reperfusion. Group II (IR group) underwent ischemia and reperfusion and group III (hesperetin group) received a $50 \mathrm{mg} / \mathrm{kg}$ dose of hesperetin intraperitoneally (IP) according to (10). Group IV (DMSO group, DMSO is the solvent of hesperetin) . To investigate its interference in this study and the effect of solvent on renal tissues, one group received $1.5 \mathrm{mg} /$ $\mathrm{kg}$ of DMSO (IP) according to (10), and finally, group $\mathrm{V}$ (IR+ hesperetin group) was exposed to ischemia and reperfusion and received hesperetin $(50 \mathrm{mg} / \mathrm{kg}$, IP) prior to reperfusion.

\section{Anesthesia Induction}

For anesthesia, 10\% ketamine hydrochloride $(50 \mathrm{mg} /$ $\mathrm{kg}$ ) was used with $2 \%$ xylazine hydrochloride $(10 \mathrm{mg} /$ $\mathrm{kg}$ ) administered intramuscularly although more doses were given if necessary during the surgical procedure. Following the administration of anesthesia, the animals were given heparin $(250 \mathrm{IU})$ through their jugular veins as a preventative measure against clotting (11).
Experimental Protocol

All animals were put on a surgical table in the dorsal recumbency position and an adhesive tape was used to immobilize their limbs. Next, a sterile technique was applied to make a skin incision on the medial surface of the left hindlimb. The femoral artery and vein were then isolated from the surrounding tissues thus exposing the femoral artery. The skeletal muscle IR was induced through clamping the femoral artery. Throughout the ischemic period, the animals were kept under anesthesia and in the dorsal recumbency position. Using a heating pad, the body temperature of the animals was kept constant and monitored via a rectal thermometer. The vascular forceps were taken off after 2 hours of ischemia and the surgical site was closed through standard procedures.

Following 24 hours of reperfusion, another dose of anesthesia was administered and then the blood sample was taken directly from the heart using sterile syringes. Next, the samples were drawn without an anticoagulation agent and then centrifuged for ten minutes at $3000 \mathrm{rpm}$ at room temperature so as to separate the serum. The serum samples were then kept at $-20^{\circ} \mathrm{C}$ in order to conduct further biochemical analyses. The renal tissue samples were collected after the animals were euthanized humanely. At which point, the collected specimens were either placed in $10 \%$ formalin or embedded inside paraffin for histological research.

\section{Measurement of Serum Urea and Creatinine}

The serum urea and creatinine concentrations were determined by using an Abbott-Aeroset autoanalyzer and their original kits (Pars Azmun Chemical Company, Iran).

\section{Biochemical Parameter Measurements}

The concentration of malondialdehyde (MDA) was measured (18) in accordance with the method proposed by Yoshioka et al (12). The levels of glutathione peroxidase (GPx) were established following the method set by Matkovics (13). The activity of superoxide dismutase (SOD) was ascertained by using both xanthine and nitroblue tetrazolium as substrates and calculating the inhibition percentage of formazan production (14). The MDA, SOD, and GPx activities were identified using enzyme assay kits (Navand Chemical Company, Iran) according to the protocols.

\section{Histopathological Analysis}

The renal tissue samples were placed in a solution of $10 \%$ formalin in the blocks of paraffin and prepared by cutting the sections of $4 \mu \mathrm{m}$. Based on the standard protocol, the samples were stained with hematoxylin and eosin (H\&E). A pathologist, who was blind to the experimental procedure, scored the amount of tissue damage. According to the protocol proposed by Chatterjee et al (Table 1), the renal injuries secondary to skeletal muscle IR were classified into four distinct grades (15). Next, five slides 
Table 1. Histological Grading According to the Protocol of Chatterje et al

\begin{tabular}{ll}
\hline Grade $\mathbf{0}$ & No Diagnostic Change \\
\hline Grade $\mathbf{1}$ & $\begin{array}{l}\text { Tubular cell swelling, brush border loss, and nuclear } \\
\text { condensation with up to } 1 / 3 \text { of the tubular profile showing } \\
\text { nuclear loss }\end{array}$ \\
\hline Grade 2 & $\begin{array}{l}\text { Is similar to grade } 1 \text {, but greater than } 1 / 3 \text { and less than } 2 / 3 \text { of } \\
\text { the tubular profile representing nuclear loss }\end{array}$ \\
\hline Grade 3 & Greater than $2 / 3$ of tubular profile indicating nuclear loss \\
\hline
\end{tabular}

from each of the tissue samples were randomly screened, and the mean value was used to represent the value of that samples.

\section{Statistical Analysis}

Data were analyzed using SPSS software, version 18. A Kolmogorov-Smirnov test was applied to determine the normal distribution of data. A comparison of the quantitative variables with a normal distribution was conducted using a Tukey post hoc test and one-way analysis of variance (ANOVA). The Kruskal-Wallis test, as the non-parametric equivalent of ANOVA, was utilized for quantitative variables that did not have a normal distribution. The study results are noted as mean \pm standard deviation, and a $P$ value of $<0.05$ was considered significant.

\section{Results}

The experimental procedures were well-tolerated by the rats, and none of them died during the experiments.

\section{Serum Levels of Urea and Creatinine}

The serum urea and creatinine levels were measured to assess the impact of hesperetin on remote renal injuries. Compared to other groups, rats with skeletal muscle IR exhibited significantly higher urea and creatinine serum levels $(P<0.05)$. The urea and creatinine levels decreased significantly following treatment with hesperetin (Table 2).

\section{Serum Biochemical Parameters}

Table 3 presents the concentrations of SOD, MDA, and GPx observed in the serum samples of both sham and experimental groups. As shown, the serum level of MDA present in the IR group was significantly greater compared with that of the sham group $(P<0.05)$. In the IR group, hesperetin could significantly ameliorate the increased expression of MDA. The antioxidant enzyme activity levels were measured as well (Table 3). Compared to the sham group, the tissue activities of SOD and GPx observed in the IR group were significantly less $(P<0.05)$. In contrast, hesperetin could significantly increase the tissue activities of both SOD and GPx $(P<0.05)$.

Histological Results

Histological changes observed in the renal tissue
Table 2. Effect of Hesperetin on the Serum Levels of Urea and Creatinine

\begin{tabular}{lcc}
\hline Group & Urea $(\mathbf{m g} / \mathbf{d L})$ & Creatinine $(\mathbf{m g} / \mathbf{d L})$ \\
\hline Sham & $38.79 \pm 7.02$ & $0.49 \pm 0.03$ \\
Ischemia reperfusion & $157.50 \pm 21.11^{*}$ & $2.63 \pm 0.11^{*}$ \\
Hesperetin & $39.3 \pm 9.09$ & $0.51 \pm 0.10$ \\
Dimethyl sulfoxide & $43.13 \pm 9.15$ & $0.56 \pm 0.09$ \\
Ischemia reperfusion + hesperetin & $51.15 \pm 8.35$ & $0.63 \pm 0.05$ \\
\hline
\end{tabular}

Note. Values are expressed as mean \pm standard deviation; *Significantly different from the other groups at $P<0.05$.

Table 3. Effect of Hesperetin on the Serum Values of MDA, SOD, and GPx

\begin{tabular}{llll}
\hline Group & MDA $(\mathbf{m m o l} / \mathbf{L})$ & SOD $(\mathbf{U} / \mathbf{m L})$ & GPx $(\mathbf{U} / \mathbf{m L})$ \\
\hline Sham & $17.90 \pm 0.65$ & $15.85 \pm 0.18$ & $0.32 \pm 0.01$ \\
Ischemia reperfusion & $26.32 \pm 0.50^{*}$ & $10.68 \pm 0.09^{*}$ & $0.26 \pm 0.02^{*}$ \\
Hesperetin & $17.72 \pm 0.09$ & $15.51 \pm 0.38$ & $0.31 \pm 0.01$ \\
$\begin{array}{l}\text { Dimethyl sulfoxide } \\
\begin{array}{l}\text { Ischemia reperfusion }+ \\
\text { hesperetin }\end{array}\end{array}$ & $19.01 \pm 0.50$ & $14.70 \pm 0.06$ & $0.30 \pm 0.00$ \\
\hline
\end{tabular}

SOD, superoxide dismutase; MDA, malondialdehyde; GPx, glutathione peroxidase.

Note. Values are expressed as mean \pm standard deviation; *Significantly different from the other groups at $P<0.05$.

Table 4. Histological Renal Injury Scores in the Experimental Groups

\begin{tabular}{lc}
\hline Group & Histological Scores \\
\hline Sham & $0.20 \pm 0.15$ \\
Ischemia reperfusion & $2.40 \pm 0.31^{*}$ \\
Hesperetin & $0.25 \pm 0.09$ \\
Dimethyl sulfoxide & $0.39 \pm 0.08$ \\
Ischemia reperfusion + hesperetin & $0.45 \pm 0.11$ \\
\hline
\end{tabular}

Note. Values are expressed as mean \pm standard deviation; *Significantly different from the other groups at $P<0.05$.

samples are summarized in Table 4. The histological analysis of sham and hesperetin groups did not show any renal morphological changes (Figures $1 \mathrm{~A}$ and $1 \mathrm{C}$ ). Contrarily, the renal tissues of the IR group demonstrated glomerular fibrosis, degeneration and necrosis of the tubular epithelium, protein casts, interstitial edema, and inflammation (Figure 1B). The degree of renal injury significantly improved in hesperetin-treated rats. Based on histological examinations, the IR group experienced higher amounts of renal changes compared to the other study groups.

\section{Discussion}

Flavonoids are powerful antioxidant agents and evidence suggests that they protect bodily tissues against oxidative stress. Studies have also reported that flavonoids may be beneficial in diseases that are attributed to oxidative stress. Hesperetin is a flavanone glycoside found in citrus fruits and can exert antioxidant activities (9). Several studies 

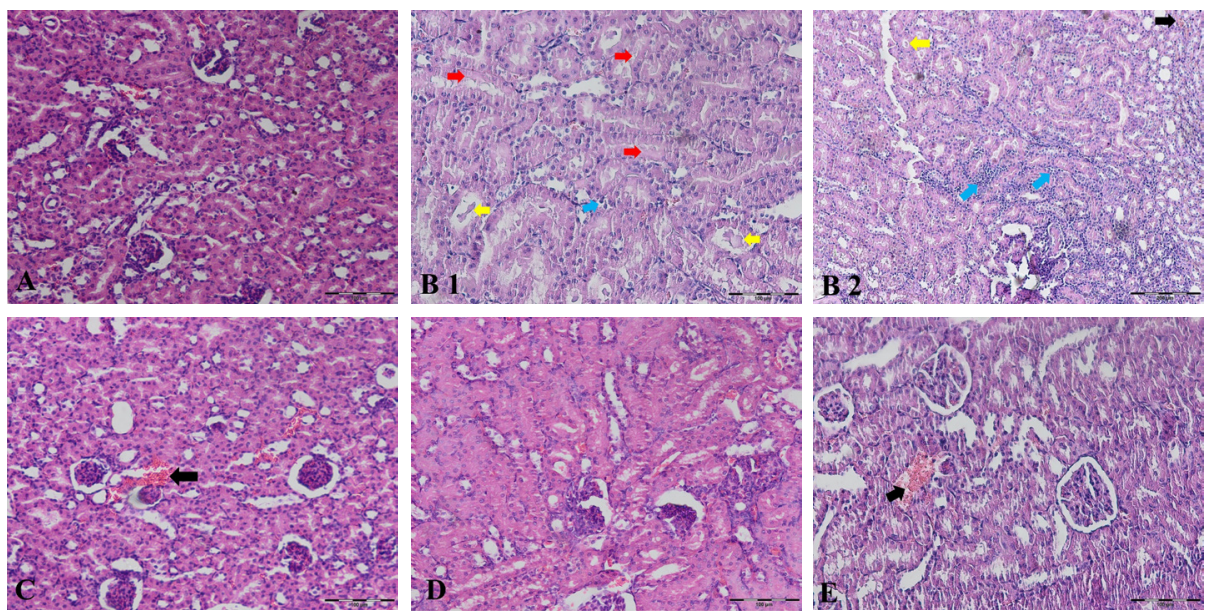

Figure 1. Histological Sections of Rat Kidney Stained With H\&E: (A) Sham group, (B1- B2) Ischemia reperfusion group, (C) Hesperetin group, (D) Dimethyl sulfoxide group, and (E) Ischemia reperfusion + Hesperetin group.

Note. H\&E: Hematoxylin and eosin; Histological analysis of the sham and hesperetin groups did not show any renal morphological changes (A and C). In contrast, the renal tissues of the ischemia reperfusion group demonstrated glomerular fibrosis and congestion (black arrow), degeneration and necrosis of the tubular epithelium (red arrow), protein casts (yellow arrow), and inflammation (blue arrow, B1-B2) with the magnification of A, B1, C, D, and E, x200, as well as B2, x100).

have focused on the effects of other substances as an antioxidant on renal remote organ injury. For instance, vitamin C (16), curcumin (17), and melatonin (18) protect kidneys from skeletal muscle IR.

Previous studies have provided evidence that hesperetin can reduce reactive oxygen species (ROS) in $\operatorname{IR}(9,19)$ in addition to preventing ischemic damage. They have demonstrated its beneficial effects on cerebral (19), cardiac (20), ovarian (10), and intestinal (21) IR models.

Ischemia can cause varying degrees of injury, the extent of which can depend on the cellular type, susceptibility to trauma, metabolism, and blood supply. The skeletal muscle tissue is highly sensitive to ischemia. In fact, it is common to observe skeletal muscle IR following burns, traumas, and surgical procedures. Even in cases where the acute ischemia is eliminated and reperfusion is restored, the potential for morbidity and mortality remains due to remote organ dysfunction (1). Based on experimental and clinical research, it is known that kidneys are greatly impacted in remote IR injury. A research study on this subject reported a correlation between post-operation renal failure and intra-operative limb ischemia (4). The importance of skeletal muscle IR in renal changes has been also highlighted in other studies $(3,22)$.

In the present study, it was expected to observe renal dysfunction and histological lesions. In the IR group, serum urea and creatinine concentrations significantly increased, which were attributed to the deterioration of renal function. The obtained results in the current study are in line with those of earlier research (3). Our results provided evidence that skeletal muscle IR leads to a systemic response within 24 hours of reperfusion, as represented by the histological impairment of the kidneys. The histopathological profile of the kidneys in the IR group revealed damaged tubules and glomerular necrosis.
However, hesperetin could diminish these changes.

Moreover, the rats exposed to skeletal muscle IR exhibited an increase in oxidative stress products including serum MDA. Lipid peroxidation resulted in both structural and functional cellular alterations, which was the maximum damage inflicted by free radicals. Cellular structures having membranes rich in unsaturated fatty acids are particularly susceptible to attack by free radicals. Consequently, a higher level of plasma MDA was observed in the IR group compared to the sham group, which could be probably attributed to greater oxidative metabolic activity and the increased concentrations of readily oxidizable polyunsaturated fatty acids present in the membrane. Antioxidants like GPx and SOD are part of the primary defense system. Specifically, these enzymes result in ROS being converted into a less reactive species (23). Significantly lower antioxidant enzyme activity was found in the IR group compared to the sham group whereas hesperetin significantly increased SOD and GPX tissue activity.

The main limitation in our research is that only one dose of hesperetin was studied, thus further studies are required to evaluate the effects of this drug in various doses. It is also recommended that the possible side effects of hesperetin on tissues be investigated by toxicological evaluations. In addition, serum hesperetin concentration was not measured in this study. Given that part of the effect of hesperetin may be due to its metabolites, it is necessary that such measurements be made in subsequent studies.

In conclusion, the current investigation demonstrates the protective capabilities of hesperetin against damage to the kidneys, as remote organs, were confirmed following skeletal muscle IR. The results indicated that by reducing oxidative stress, hesperetin might help protect from remote organ injury due to IR. Therefore, hesperetin 
could be used as an adjunctive therapeutic agent in cases of supposed remote organ IR injury.

\section{Authors' Contribution}

PM, AA and FM: concept and design. HAT: data collection and interpretation of the data. HAT and PM: performing of the study and writing of the draft. All authors read and approved the study.

\section{Conflict of Interests}

The authors declare that there is no conflict of interests.

\section{Ethical Issues}

This study was conducted in accordance with the Animal Ethics Committee guidelines set out by the Islamic Azad University (No: 2019-7/0103).

\section{Financial Support}

None.

\section{References}

1. Kuroda Y, Togashi H, Uchida T, Haga K, Yamashita A, Sadahiro M. Oxidative stress evaluation of skeletal muscle in ischemiareperfusion injury using enhanced magnetic resonance imaging. Sci Rep. 2020;10(1):10863. doi:10.1038/s41598020-67336-4

2. de Oliveira Armstrong DM, da Costa Armstrong A, Figueiredo $\mathrm{RC}$, et al. Sildenafil citrate protects skeletal muscle of ischemia-reperfusion injury: immunohistochemical study in rat model. Acta Cir Bras. 2013;28(4):282-287. doi:10.1590/ s0102-86502013000400008

3. Emrecan B, Tulukoglu E, Bozok S, et al. Effects of Iloprost and pentoxifylline on renal ischemia-reperfusion in rabbit model. Eur J Med Res. 2006;11(7):295-299.

4. Gyurkovics E, Aranyi P, Stangl R, et al. Postconditioning of the lower limb--protection against the reperfusion syndrome. J Surg Res. 2011;169(1):139-147. doi:10.1016/j.jss.2009.10.014

5. Rhee MH, Park HJ, Cho JY. Salicornia herbacea: botanical, chemical and pharmacological review of halophyte marsh plant. J Med Plants Res. 2009;3(8):548-555. doi:10.5897/ jmpr.9001099

6. Panche AN, Diwan AD, Chandra SR. Flavonoids: an overview. J Nutr Sci. 2016;5:e47. doi:10.1017/jns.2016.41

7. Ma C, Gao W, Gao Y, Man S, Huang L, Liu C. Identification of chemical constituents in extracts and rat plasma from Fructus Aurantii by UPLC-PDA-Q-TOF/MS. Phytochem Anal. 2011;22(2):112-118. doi:10.1002/pca.1252

8. Li C, Schluesener H. Health-promoting effects of the citrus flavanone hesperidin. Crit Rev Food Sci Nutr. 2017;57(3):613631. doi:10.1080/10408398.2014.906382

9. Roohbakhsh A, Parhiz H, Soltani F, Rezaee R, Iranshahi M. Molecular mechanisms behind the biological effects of hesperidin and hesperetin for the prevention of cancer and cardiovascular diseases. Life Sci. 2015;124:64-74. doi:10.1016/j.Ifs.2014.12.030

10. Cakir Gungor AN, Gencer M, Karaca T, et al. The effect of hesperetin on ischemia-reperfusion injury in rat ovary. Arch Gynecol Obstet. 2014;290(4):763-769. doi:10.1007/s00404014-3267-8

11. Ashrafzadeh Takhtfooladi M, Jahanshahi A, Sotoudeh A, Jahanshahi G, Ashrafzadeh Takhtfooladi H, Aslani K. Effect of tramadol on lung injury induced by skeletal muscle ischemia-reperfusion: an experimental study. J Bras Pneumol. 2013;39(4):434-439. doi:10.1590/s180637132013000400006

12. Yoshioka T, Kawada K, Shimada T, Mori M. Lipid peroxidation in maternal and cord blood and protective mechanism against activated-oxygen toxicity in the blood. Am J Obstet Gynecol. 1979;135(3):372-376. doi:10.1016/0002-9378(79)90708-7

13. Matkovics B. Determination of enzyme activity in lipid peroxidation and glutathione pathways. Laboratoriumi Diagnosztika. 1988;15:248-250.

14. Sun Y, Oberley LW, Li Y. A simple method for clinical assay of superoxide dismutase. Clin Chem. 1988;34(3):497-500. doi:10.1093/clinchem/34.3.497

15. Chatterjee PK, Patel NS, Kvale EO, et al. Inhibition of inducible nitric oxide synthase reduces renal ischemia/reperfusion injury. Kidney Int. 2002;61(3):862-871. doi:10.1046/j.15231755.2002.00234.x

16. Ozcan AV, Sacar M, Aybek $H$, et al. The effects of iloprost and vitamin $\mathrm{C}$ on kidney as a remote organ after ischemia/ reperfusion of lower extremities. J Surg Res. 2007;140(1):2026. doi:10.1016/j.jss.2006.04.031

17. Karahan MA, Yalcin S, Aydogan $\mathrm{H}$, et al. Curcumin and dexmedetomidine prevents oxidative stress and renal injury in hind limb ischemia/reperfusion injury in a rat model. Ren Fail. 2016;38(5):693-698. doi:10.3109/0886022x.2016.1157746

18. Kaçmaz A, User EY, Sehirli AO, Tilki M, Ozkan S, Sener G. Protective effect of melatonin against ischemia/reperfusioninduced oxidative remote organ injury in the rat. Surg Today. 2005;35(9):744-750. doi:10.1007/s00595-005-3027-2

19. Gaur V, Kumar A. Hesperidin pre-treatment attenuates NO-mediated cerebral ischemic reperfusion injury and memory dysfunction. Pharmacol Rep. 2010;62(4):635-648. doi:10.1016/s1734-1140(10)70321-2

20. Selvaraj P, Pugalendi KV. Hesperidin, a flavanone glycoside, on lipid peroxidation and antioxidant status in experimental myocardial ischemic rats. Redox Rep. 2010;15(5):217-223. doi:10.1179/135100010x12826446921509

21. Pehlivan M, Hazinedaroglu SM, Kayaoglu HA, et al. The effect of diosmin hesperidin on intestinal ischaemia--reperfusion injury. Acta Chir Belg. 2004;104(6):715-718. doi:10.1080/00 015458.2004.11679649

22. Teruya R, Fagundes DJ, Oshima CT, et al. The effects of pentoxifylline into the kidneys of rats in a model of unilateral hindlimb ischemia/reperfusion injury. Acta Cir Bras. 2008;23(1):29-35. doi:10.1590/s0102-86502008000100006

23. Stark AN, Jackson G, Carey PJ, Arfeen S, Proctor SJ. Severe renal toxicity due to intermediate-dose methotrexate. Cancer Chemother Pharmacol. 1989;24(4):243-245. doi:10.1007/ bf00257626

Copyright $\odot 2022$ The Author(s); This is an open-access article distributed under the terms of the Creative Commons Attribution License (http://creativecommons.org/licenses/by/4.0), which permits unrestricted use, distribution, and reproduction in any medium, provided the original work is properly cited. 